\title{
Emergent Cardiac Anomaly Classification Using Cascaded Auto- associative Multilayer Perceptrons for Bio-healthcare Systems
}

\author{
Hye-Jin Lee, Jaeho Oh, Chang-Beom Kwon and Sang-Woo Ban* \\ Dept. of Information and Communication Engineering, Dongguk University, \\ 123 Dongdae-ro, Gyeongju, Gyeongbuk 780-714, Republic of Korea \\ *Corresponding author: swban@dongguk.ac.kr
}

\begin{abstract}
Proper indication of emergent cardiac anomalies is essential to saving human lives. Electrocardiogram (ECG) signals are mainly considered for indicating cardiac status. This work proposes a model that discriminates emergent cardiac anomalies (e.g. ventricular tachyarrhythmia, congestive heart failure, malignant ventricular ectopy, supraventricular arrhythmia) from normal cardiac status using an artificial neural network. The histogram of gradient (HOG) and principal component analysis (PCA) are applied to extract generic features of the ECG signals. Five auto-associative multilayer perceptrons (AAMLP) concatenated in a cascade manner are proposed for classification of four emergent cardiac ECG signals and normal ECG signals, which was developed for implementing a primitive prototype for a mobile bio-healthcare system. Experimental results show that the proposed model successfully classifies emergent cardiac anomalies.
\end{abstract}

Keywords: Emergent cardiac anomalies, ECG signals, HOG, PCA, cascaded AAMLP, mobile bio-healthcare system

\section{Introduction}

Emergent cardiac anomalies are very serious for human being. Rapid and proper indication of occurrence of emergent cardiac anomalies is a very essential process for saving human being from death [1]. Arrhythmia is the symptoms that atrial beats becomes faster or slower due to abnormality in an electric signal path of the heart. The symptoms of arrhythmia can vary depending on the type of arrhythmia, which usually feel get along without any symptoms. Supraventricular arrhythmia refers to severe arrhythmias that have appeared more than 10 times per minute or a heart rate more than 150 times to 200 times per minute, which can cause sudden death if it is very severe. Therefore early indication of emergent cardiac anomalies is very important [1].

Most of the algorithms processing electrocardiogram (ECG) signals focus on indicating a specific feature for diagnosis, which works well in many applications [2-11]. However, in some cases, it is very hard to find an essential characteristic feature indicating occurrence of an abnormal symptom. Therefore, it is necessary to develop a generic feature-extraction method for monitoring any emergency situation by detecting abnormal ECG signals having different characteristic features compared with ECG signals obtained during normal states. Analysis of ECG signals is of great importance in the detection of cardiac anomalies. Many non-linear approaches and time-frequency approaches are introduced for ECG signal processing for providing stable and effective rhythm detection [2-9]. Oswoski et al., [9] proposed a hierarchical system that combines a multilayer perceptron (MLP) and another artificial neural network. Woo et al., [10] proposed an ECG signal analysis model using one-class support vector machine to discriminate superaventricular arrhythmia as an abnormal ECG signal from normal ECG signals. Lee et al., [11] also proposed an ECG classification model using auto-associative multilayer 
perceptrons (AAMLP) to discriminate three abnormal cases from a normal case.

In order to efficiently analyze the patterns of ECG signals, a general feature-extraction method and an associative neural network model are considered. As a generic feature extraction approach, the histogram of gradient (HOG) of ECG signals is considered, which is robust in properly extracting characteristics of general ECG signals [10, 11]. As well, projections on mainly contributive principal components of HOG were also utilized as additional features that can represent more highly intrinsic features of ECG signals. In order to efficiently discriminate and recognize ECG signals using the extracted features, this study considers AAMLP, which is known to show proper performance with a partially exposed problem such as ECG signal classification. Multiple AAMLPs are organized in a cascade manner to enhance classification performance on ECG signals of four emergent cardiac anomalies. The proposed system shows plausible classification performance by properly discriminating ECG signals caused by four abnormal symptoms (e.g. congestive heart failure, malignant ventricular ectopy, ventricular tachyarrhythmia, supraventricular arrhythmia) from normal ECG signals. Moreover a prototype of a mobile platform for ECG monitoring was implemented in order to apply to a u-healthcare system in a future. The ECG signals utilized in the experiments were obtained from the Physionet database [12-16].

Section 2 describes the proposed ECG signal classification model including the proposed feature extraction method and the mobile U-healthcare embedded system for ECG signal monitoring. Section 3 describes the experiments and the conclusion and discussion will be followed in Section 4.

\section{Proposed ECG Signal Classification Model}

Figure 1 is the procedure of the proposed ECG classification model in this paper, which mainly consists of two parts. One is generic feature extraction from the input ECG signals, and the other is classification implemented by five AAMLPs organized in a cascade manner. The extracted features are used as input for AAMLP. The first AAMLP decides whether an input ECG signal is normal or abnormal. The subsequent AAMLPs, applied in a cascade manner, are utilized for deciding a specific symptom of the input EEG signals, in which each AAMLP plays a role for classifying the input signal into two classes such as a trained class and a non-trained class. Thus, five AAMLPs are trained using normal signals, congestive heart failure signals, ventricular tachyarrhythmia, malignant ventricular ectopy, and supraventricular arrhythmia. Most ECG signal classification problems might be considered as a partially exposed problem. Therefore, AAMLP can be an appropriate choice for an ECG classification model. According to the experiments, a cascade of classification models shows more plausible classification performance. As a result, in the proposed ECG classification model, a cascaded AAMLP model is considered.

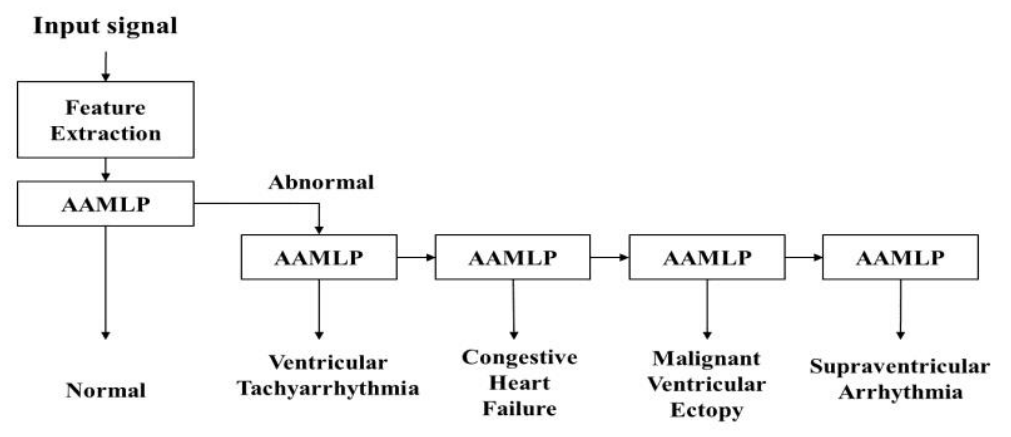

Figure 1. Proposed ECG Classification Model 


\subsection{Feature extraction of ECG signals}

Many approaches have been considered for feature extraction from ECG signals. This paper adopted the HOG to extract as generic features of ECG signals. HOG is utilized to appropriately represent a generic wave pattern of each specific ECG signal [11]. A human expert can discriminate each different ECG wave pattern caused by different symptoms without any critical analysis of those ECG signals in detail, since each different symptom generates its specific ECG signal pattern. Thus, HOG can be considered for proper features to discriminate each specific ECG signal from other ECG signals having different characteristics, in a manner similar to a human expert. Moreover, a human expert can capture intrinsic features having higher level characteristics of ECG signals. In order to mimic this kind of extraction of high-level intrinsic features of ECG signals, the proposed model considered principal component analysis (PCA). Principle components are obtained via PCA using HOG of the ECG signals. Thus, in the proposed model, each ECG feature is represented by both $\mathrm{HOG}$ and projections in mainly contributive principal components obtained via PCA. Each extracted HOG and its projections onto principal components are used as an input to a classification model.

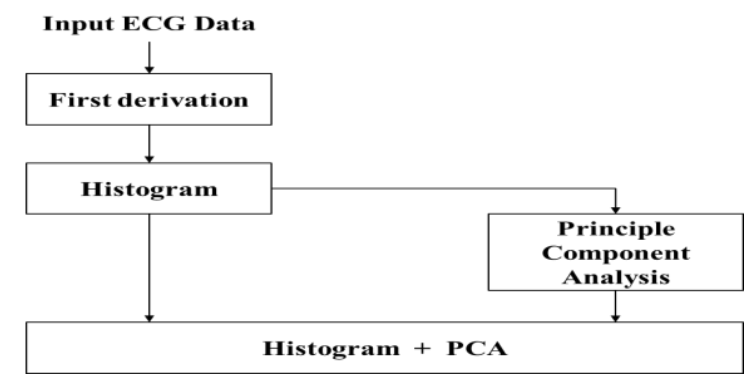

Figure 2. Feature Extraction Procedure of Proposed Model

In order to obtain HOG, firstly a gradient of ECG signals is obtained using the $1^{s t}$ derivative of ECG signals. The $1^{\text {st }}$ derivative of input ECG signal is calculated by Eq. (1):

$g(t)=$ raw $\_$signal $(t+\Delta t)-$ raw _ signal $(t)$, where $\Delta t$ is a sampling time

where raw _ signal $(t)$ is an amplitude of raw input ECG signal at time $t$. The gradient feature obtained by the $l^{\text {st }}$ derivative of input signal can generically represent temporal changing patterns of time-series signals. Therefore it is natural that the $1^{s t}$ derivative of input signals can be used for describing generic temporal patterns of ECG signals. By applying a histogram approach, statistical characteristics of temporal features of ECG signals can be considered. For generalization, before obtaining a histogram, normalization of signal amplitudes is processed by Eq. (2) since input ECG signals have variously scaled amplitude levels:

$\bar{g}(t)=\frac{g(t)}{\max \{|g(t)|\} \text { for } \forall t}$, where $|g(t)|$ is an absolute value of $g(t)$

Then quantization of the normalized $l^{s t}$ derivative signals is computed by Eq. (3) before obtaining the histogram of the normalized $l^{\text {st }}$ derivate signals, which makes every histogram features have the same dimension. 


$$
q(t)=\left(\frac{\max _{-} \bar{g}-\bar{g}(t)}{\max _{-} \bar{g}-\min _{-} \bar{g}} \times 100\right) \bmod N
$$

where max $\bar{g}_{-}$and $\min _{-} \bar{g}$ are maximum and minimum values for all $g(t)$, respectively. And $N$ is the number of quantization levels for generating a histogram. In the proposed model, $N$ was experimentally chosen as 35 . Finally, a histogram of gradient of ECG signal is obtained by Eq. (4):

$H O G(i)=\#$ of $q(t)$, where $q(t)=i, 0 \leq i \leq N-1$

This obtained histogram is used as a feature of each ECG signal having robust characteristic against noise signals.

In the proposed model, PCA also considered for extracting higher level of features. PCA seeks a projection that best represents the data in a least-squares sense, in which PCA projects $d$-dimension data onto a lower-dimension subspace in a way that is optimal in a sum-squared error sense [17]. First, the $d$-dimension mean vector $\boldsymbol{\mu}$ and $d \times d$ covariance matrix $\boldsymbol{\Sigma}$ are computed for the full training data set of HOGs of ECG signals. Next, the eigenvectors $\mathbf{e}_{i}$ and eigenvalues $\lambda_{i}$ are computed, and sorted according to decreasing eigenvalue.

$\lambda_{1}>\lambda_{2}>\cdots>\lambda_{d}$

The $k$ eigenvectors having the largest eigenvalues are chosen as mainly contributive principal components. By projecting each $d$-dimension data onto the chosen $k$ eigenvectors, $k$-dimension representation is generated for each $d$-dimension data. In the proposed model, $d$ is 35 , which is the same as the histogram level $N$ in Eq. (3) and $k$ was chosen as 3 by performance analysis according to a varying number of principal components. In the proposed model, three-dimension data obtained by projections of each 35-dimension HOG onto three chosen principal components are considered as high-level feature of HOG, since lower dimensional projection onto principal components can represent abstract feature of original data space having much higher dimension.

Therefore, each feature of each ECG signal is represented by a 38-dimension vector, in which 35-dimension data come from a 35-dimension HOG and threedimension data come from projecting the 35-dimension HOG onto three principal components.

\subsection{Classification of ECG signals using cascaded AAMLPs}

ECG signals have much within-class variability. Such an ECG classification problem might be one of the partially-exposed environments problems, which are well known problems where training data from one-class is very little or nonexistent $[11,18]$. AAMLP has been successfully used in many such partiallyexposed environments [18]. The proposed model uses an AAMLP with 4-layers, which are mapping layer, bottleneck layer, de-mapping layer, and output layer. Dimension reduction is achieved by projecting the vectors in a subspace captured by the set of weights in the bottleneck layer through the mapping layer. Dimension expansion is achieved by mapping the lower dimension vectors onto a hyper-surface captured by the set of weights in the output layer through the de-mapping layer. An AAMLP is basically a neural network whose input and target vectors are the same 
[18]. The training process of an AAMLP is the same as that of a multi-layer perceptron (MLP). After successful training, each input data is classified by comparing similarity between an input vector and a corresponding output vector of the AAMLP. Figure 3 shows a 4-layer AAMLP applied in the proposed model.

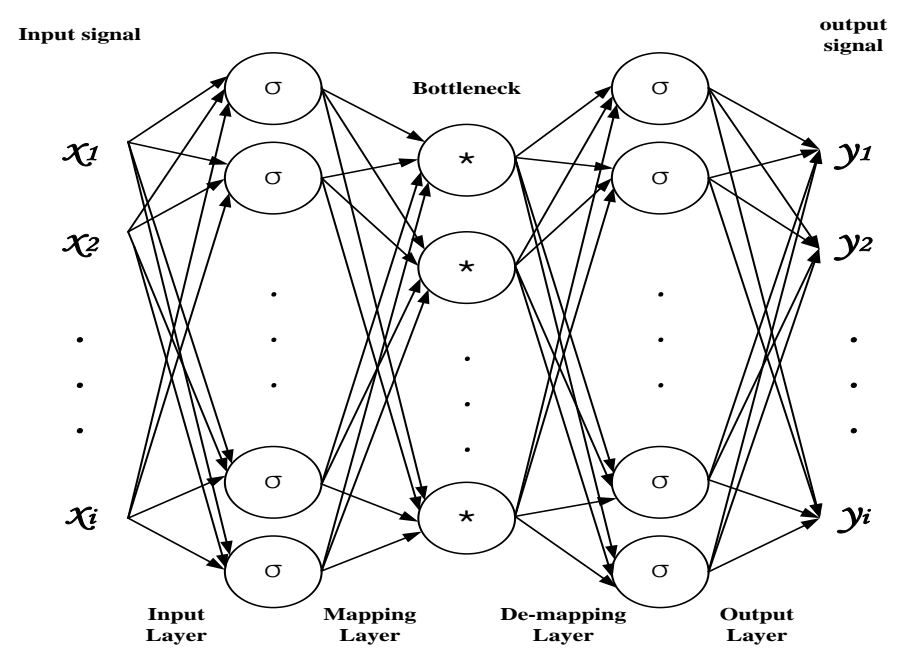

Figure 3. Architecture of 4-layers AAMLP

The proposed model uses a 38-dimension vector obtained by the HOG of input ECG signals and its projections onto principal components as an input data for the AAMLP. Let $F$ denote an auto-associative mapping function, and $x_{i}$ and $y_{i}$ indicate an input and output vector, respectively. Then the function $F$ is usually trained to minimize the following mean square error given by Eq. (6).

$E=\sum_{i=1}^{n}\left\|x_{i}-y_{i}\right\|^{2}=\sum_{i=1}^{n}\left\|x_{i}-F\left(x_{i}\right)\right\|^{2}$

where $n$ denotes the number of output nodes and $n$ is 38 in the proposed model.

After the training process is successfully finished, every feature extracted from input ECG signal is used for an input of AAMLP, and the similarity of input and output of AAMLP is measured. If the similarity value is above a threshold, the input is regarded as a trained class signal. Or, the input is regarded as an untrained class signal as shown in Eq. (7). The degree of similarity $E$ in Eq. (7) is also calculated by Eq. (6).

input ECG signal is classified as $\left\{\begin{array}{l}\text { a trained class if } E \leq \theta \\ a \text { non-trained class if } E>\theta\end{array}\right\}$

\subsection{Bio-healthcare Monitoring System}

The proposed emergent ECG signal classification model is aimed to apply to a ubiquitous bio-healthcare system which was partly implemented on S3C 6400 mobile embedded platform with ARM 11 processor $[11,19]$. The proposed bio-healthcare system uses ZigBee communication based on wireless sensor network. A wireless ECG sensor node plays a role for sensing ECG signals and wirelessly transmitting sensed ECG signals to one mobile embedded platform in every $256 \mathrm{~ms}$ [11]. Each radio communication is conducted by $250 \mathrm{kbps}$ transmission rate using a $2.4 \mathrm{GHz}$ frequency band by the CC2420 
wireless communication chip [11]. In addition, one mobile embedded platform receives ECG signals from the ECG sensor node and classifies the signals. And then it sends the classification result to another mobile embedded platform, which monitors heart conditions of a human by showing recognized states of heart signals. HBE-ZigbeX motes were utilized as a wireless sensor node for the wireless sensor network for ECG signal monitoring. The HBE-ZigbeX mote basically consists of a micron controller (ATmega 128L), a wireless communication chip (CC2420), sensors and antenna [11, 20].

\section{Experimental Results}

Five different kinds of ECG signals are considered in the experiment, which consists of one category of normal ECG signals and four different categories of emergent ECG signals, such as ventricular tachyarrhythmia, congestive heart failure, malignant ventricular ectopy and supraventricular arrhythmia. ECG signals for this experiments were obtained from the Physionet database [12-16]. The sampling rate of all the ECG signals was $125 \mathrm{~Hz}$. Figures 4 and 5 show raw ECG signals with 1281 dimensions and HOG of the ECG signals with 35 dimensions. In Figure 4, the horizontal axis and the vertical axis represent sampling time and scaled amplitude, respectively. In Figure 5, the horizontal axis and the vertical axis represent quantized level and normalized frequency, respectively, of the HOG.

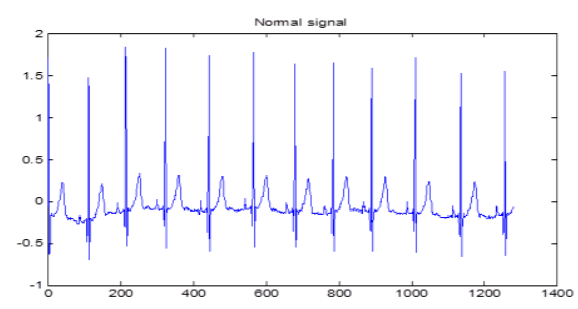

(a)

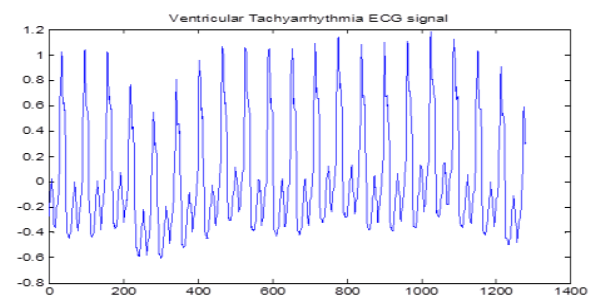

(c)

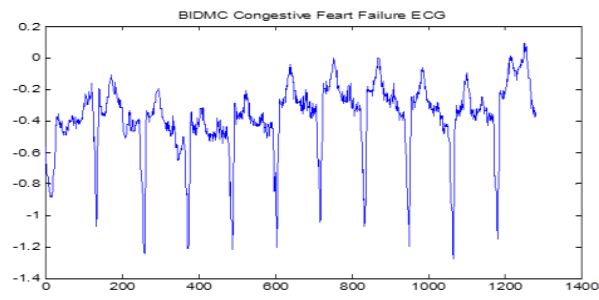

(b)

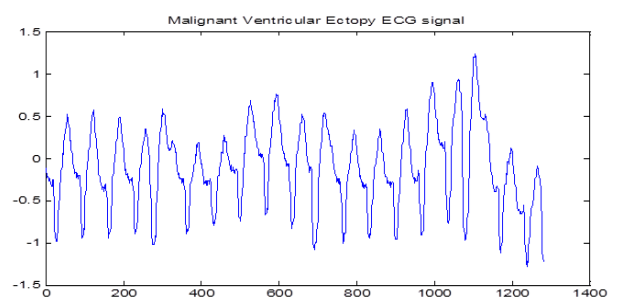

(d)

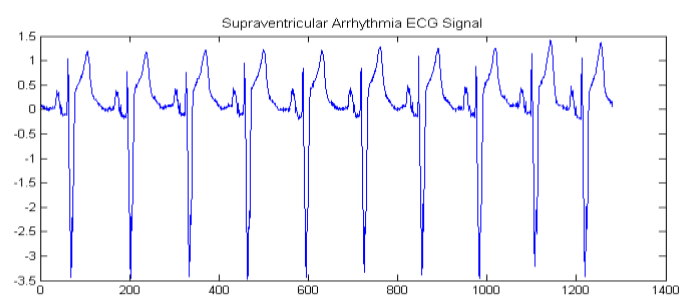

(e)

Figure 4. Raw ECG Signals; (a) Normal Signal, (b) Congestive Feart Failure Signal, (c) Ventricular Tachyarrhythmia Signal, (d) Malignant Ventricular Ectopy Signal, (e) Supraventricular Arrhythmia Signal 


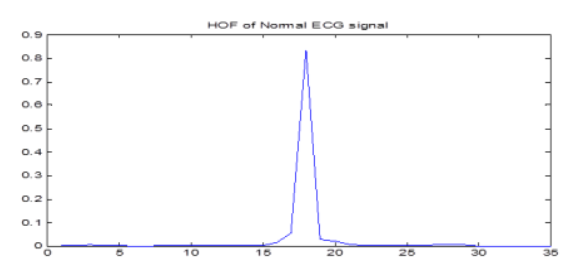

(a)

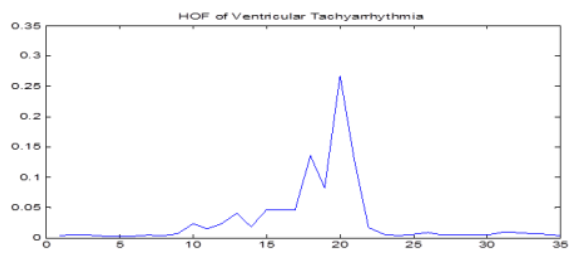

(c)

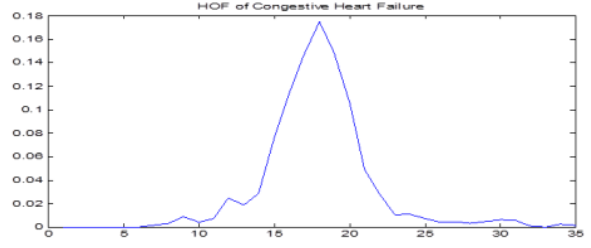

(b)

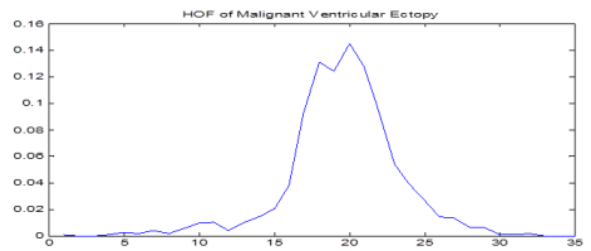

(d)

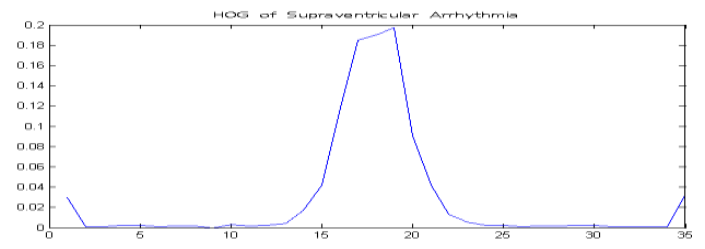

(e)

Figure 5. HOG of ECG signals; (a) HOG of Normal Signal, (b) HOG of Congestive Heart Failure Signal, (c) HOG of Ventricular Tachyarrhythmia Signal, (d) HOG of Malignant Ventricular Ectopy Signal, (e) HOG of Supraventricular Arrhythmia Signal

In this experiment, two different performance analyses from three performance analyses were conducted in order to verify the appropriateness of the feature selections. First, an experiment was conducted to obtain the optimal number of principal components, which were considered for extracting higher abstract features by projecting HOGs of raw ECG signals onto the chosen principal components. Thus, every feature of each ECG signal is represented by both the 35-dimension HOG and its projections onto the chosen principal components. In order to decide the optimal number of principal components to utilize for feature extraction, classification performance according to a varying number of principal components was compared. As shown in TABLE 1, best performance was obtained when three or four principal components were considered. Thus, in the proposed model, three main principal components are selected for feature extraction. Therefore, each ECG signal is represented by a 38-dimension vector, which is generated by concaten ating a 35-dimension HOG and a three-dimension projection vector onto three chosen principal components of a 35-dimension HOG. Second, in order to verify the reason why PCA is considered to obtain additional features, performance comparison is conducted between the proposed model considering both HOG and PCA and the model considering only HOG. As shown in TABLE 2, the proposed model shows better performance than the model considering only HOG. Therefore, a feature extraction method for the proposed ECG classification model was decided according to analysis of these two experiments. 
Table 1. Analysis of Classification Performance according to Different Number of Principal Components

\begin{tabular}{|c|c|c|c|c|c|c|}
\hline \multirow{2}{*}{$\begin{array}{l}\text { Categories of } \\
\text { ECG Signals }\end{array}$} & \multicolumn{6}{|c|}{$\begin{array}{l}\text { Classification performance according to } \\
\text { different number of principal components(PC) } \\
\text { \# of correctly classified signals / \# of total signals }\end{array}$} \\
\hline & $1^{\text {st }} \mathbf{P C}$ & $\begin{array}{c}1^{\text {st }} \sim 2^{\text {nd }} \\
\text { PCs }\end{array}$ & $\begin{array}{c}1^{\mathrm{st}} \sim 3^{\mathrm{rd}} \\
\mathrm{PCs}\end{array}$ & $\begin{array}{c}1^{\text {st }} \sim 4^{\text {th }} \\
\text { PCs }\end{array}$ & $\begin{array}{c}1^{\text {st }} \sim 5^{\text {th }} \\
\text { PCs }\end{array}$ & $\begin{array}{c}1^{\text {st }} \sim 6^{\text {th }} \\
\text { PCs }\end{array}$ \\
\hline Normal & $\begin{array}{c}15 / 15 \\
(100.0 \%)\end{array}$ & $\begin{array}{c}15 / 15 \\
(100.0 \%)\end{array}$ & $\begin{array}{c}15 / 15 \\
(100.0 \%)\end{array}$ & $\begin{array}{c}15 / 15 \\
(100.0 \%)\end{array}$ & $\begin{array}{c}15 / 15 \\
(100.0 \%)\end{array}$ & $\begin{array}{c}15 / 15 \\
(100.0 \%)\end{array}$ \\
\hline $\begin{array}{c}\text { Ventricular } \\
\text { Tachyarrhythmia }\end{array}$ & $\begin{array}{c}10 / 12 \\
(83.3 \%)\end{array}$ & $\begin{array}{c}10 / 12 \\
(83.3 \%)\end{array}$ & $\begin{array}{c}12 / 12 \\
(100.0 \%)\end{array}$ & $\begin{array}{c}12 / 12 \\
(100.0 \%)\end{array}$ & $\begin{array}{c}11 / 12 \\
(91.7 \%)\end{array}$ & $\begin{array}{c}10 / 12 \\
(83.3 \%)\end{array}$ \\
\hline $\begin{array}{l}\text { Congestive } \\
\text { Heart Faliure }\end{array}$ & $\begin{array}{c}10 / 11 \\
(90.9 \%)\end{array}$ & $\begin{array}{c}11 / 11 \\
(100.0 \%)\end{array}$ & $\begin{array}{c}10 / 11 \\
(90.9 \%)\end{array}$ & $\begin{array}{c}11 / 11 \\
(100.0 \%)\end{array}$ & $\begin{array}{c}10 / 11 \\
(90.9 \%)\end{array}$ & $\begin{array}{c}11 / 11 \\
(100.0 \%)\end{array}$ \\
\hline $\begin{array}{l}\text { Malignant } \\
\text { Ventricular } \\
\text { Ectopy }\end{array}$ & $\begin{array}{c}11 / 11 \\
(100.0 \%)\end{array}$ & $\begin{array}{c}10 / 11 \\
(90.9 \%)\end{array}$ & $\begin{array}{c}11 / 11 \\
(100.0 \%)\end{array}$ & $\begin{array}{c}10 / 11 \\
(90.9 \%)\end{array}$ & $\begin{array}{c}10 / 11 \\
(90.9 \%)\end{array}$ & $\begin{array}{c}10 / 11 \\
(90.9 \%)\end{array}$ \\
\hline $\begin{array}{c}\text { Supraventricular } \\
\text { Arrhythmia }\end{array}$ & $\begin{array}{c}19 / 25 \\
(76.0 \%)\end{array}$ & $\begin{array}{c}24 / 25 \\
(96.0 \%)\end{array}$ & $\begin{array}{c}24 / 25 \\
(96.0 \%)\end{array}$ & $\begin{array}{c}24 / 25 \\
(96.0 \%)\end{array}$ & $\begin{array}{c}24 / 25 \\
(96.0 \%)\end{array}$ & $\begin{array}{c}25 / 25 \\
(100.0 \%)\end{array}$ \\
\hline $\begin{array}{c}\text { Average } \\
\text { performance }\end{array}$ & $90.04 \%$ & $94.04 \%$ & $97.38 \%$ & $97.38 \%$ & $93.90 \%$ & $94.84 \%$ \\
\hline
\end{tabular}

Table 2. Classification Performance Comparison according to Different Features for Verifying Effectiveness of PCA Features

\begin{tabular}{|c|c|c|}
\hline \multirow{2}{*}{ Categories of ECG Signals } & \multicolumn{2}{|c|}{$\begin{array}{c}\text { Classification performance } \\
\text { \# of correctly classified signals } \\
\text { / \# of total signals }\end{array}$} \\
\cline { 2 - 3 } & $\begin{array}{c}\text { using HOG only } \\
\text { as features }\end{array}$ & $\begin{array}{c}\text { using HOG \& PCA } \\
\text { as features }\end{array}$ \\
\hline Normal & $15 / 15(100 \%)$ & $15 / 15(100 \%)$ \\
\hline Ventricular Tachyarrhythmia & $10 / 12(83.3 \%)$ & $12 / 12(100 \%)$ \\
\hline Congestive Heart Failure & $10 / 11(90.9 \%)$ & $10 / 11(90.9 \%)$ \\
\hline Malignant Ventricular Ectopy & $10 / 11(90.9 \%)$ & $11 / 11(100 \%)$ \\
\hline Supraventricular arrhythmia & $17 / 25(68.8 \%)$ & $24 / 25(96.0 \%)$ \\
\hline
\end{tabular}

Finally, performance of the proposed ECG classification model was compared with that of Woo's model [6], in which the proposed model showed better performance than Woo's model. Actually, Woo et al. [6] considered discriminating only a supraventricular arrhythmia as an abnormal ECG from a normal ECG. However, this study conducted the experiments using the same five different categories of ECG signals considered in this work for Woo's model in order to properly compare performance. As shown in TABLE 3, the proposed model shows 
plausible classification performance for five different categories of ECG signals. As well, the proposed model shows better performance than Woo's model.

Table 3. Classification Performance Comparison

\begin{tabular}{|c|c|c|}
\hline Categories of ECG Signals & $\begin{array}{c}\text { Comparison model } \\
\text { (Woo's model) }\end{array}$ & $\begin{array}{c}\text { Proposed } \\
\text { model }\end{array}$ \\
\hline Normal & $100.0 \%$ & $100.0 \%$ \\
\hline Ventricular Tachyarrhythmia & $100.0 \%$ & $100.0 \%$ \\
\hline Congestive Heart Failure & $83.0 \%$ & $90.9 \%$ \\
\hline Malignant Ventricular Ectopy & $100.0 \%$ & $100.0 \%$ \\
\hline Supraventricular arrhythmia & $92.6 \%$ & $96.0 \%$ \\
\hline
\end{tabular}

\section{Conclusion and Future Works}

An ECG signal classification model was proposed for discriminating five different categories of ECG signals, such as four different emergent categories of ECG signal and one category for the normal ECG signal. HOG and PCA were considered for generic feature extraction, and multiple AAMLPs organized in a cascade manner were applied for pattern classification. The experimental results showed that the proposed model generated plausible classification performance. The proposed model was aimed to apply to a bio-healthcare system for monitoring patients at emergency room in a hospital and patients with heart disease.

The limitation of this work was that the experiments conducted in this work utilized ECG signals obtained from the public DB. In order to make the proposed model be more realistic one working in real situations, clinical ECG signals should be considered in the future experiments by collaborating with emergency clinicians. As further works, we are also considering to develop a personalized bio-healthcare monitoring system.

\section{Acknowledgments}

This work was partly based on the paper [11] in the following references.

\section{References}

[1] G. D. Clifford, F. Azuaje and P. E. McSharry, Advanced methods and tools for ECG data analysis, Artech House Inc., (2006).

[2] U. R. Acharya, P. S. Bhat, S. S. Iyengar, A. Rao and S. Dua, "Classification of hear rate data using artificial neural network and fuzzy equivalence relation", Pattern Recognition, vol. 36, (2003), pp. 61-68.

[3] Z. Doku and T. Olmez, "ECG beat classification by a novel hybrid neural network", Computer Methods and Programs in Biomedicine, vol. 66, (2001), pp. 167-181.

[4] H. X. Zhang, Y. S. Zhu and Z. M. Wang, "Complexity measure and complexity rate information based detection of ventricular tachycardia and fibrillation", Medical \& Biological Engineering \& Computing, vol. 38, (2000), pp. 553-557.

[5] M. I. Oiws, A. H. Abou-Zied and A. M. Youssef, "Study of Features based on Nonlinear Dynamical Modeling in ECG Arrhythmia Detection and Classification", IEEE Trans. Biomedical Engineering, vol. 49, no. 7, (2002), pp. 733-736. 
[6] T. Stamkopoulos, K. Diamantaras, N. Maglaveras and M. Strintzis, "ECG Analysis Using Nonlinear PCA Neural Networks for Ischemai Detection”, IEEE Transactions on Signal Processing, vol. 46, no. 11, (1998), pp. 3058-3067.

[7] Y. H. Hu, W. J. Tompkins and Q. Xue, "Artificial Neural Network for ECG Arrhythmia Monitoring", Proceedings of International Joint Conference on Neural Networks, vol. 2, June, (1992), pp. 987-992.

[8] B. Ghoraani, S. Krishnan, R. J. Selveraj and V. S. Chauhan, "Adaptive time-frequency matrix features for $\mathrm{T}$ wave alternans analysis", Proceedings of International Conference of the IEEE EMBS Minneapolis, (2009), pp. 39-42.

[9] S. Osowski and T. H. Linh, "ECG Beat Recognition using Fuzzy Hybrid Neural Network", IEEE Trans. Biomed. Eng. vol. 48, (2001), pp. 1265-1271.

[10] S. M. Woo, H. J. Lee, B. J. Kang and S. W. Ban, "ECG signal monitoring using one-class support vector machine", Proceedings of the $9^{\text {th }}$ International Conference on Applications of Electrical Engineering", Ningbo, China, (2010), January 10-12, pp. 132-137.

[11] H. J. Lee, D. O. Kim, B. J. Kang and S. W. Ban, "Mobile embedded health-care system working on wireless sensor network," Proceedings of the 3rd International Conference on Communications and Mobile Computing, Qingdao, China, (2011), April 18-20, pp. 161-164.

[12] http://physionet.org/physiobank/database/nsrdb/, The MIT-BIH Normal Sinus Rhythm Database.

[13] http://physionet.org/physiobank/database/chfdb/, The BIDMC Congestive Heart Failure Database.

[14] http://physionet.org/physiobank/database/vfdb/, The MIT-BIH Malignant Ventricular Arrhythmia Database.

[15] http://physionet.org/physiobank/database/cudb/, Creighton University Ventricular Tachyarrhythmia Database.

[16] http://www.physionet.org/physiobank/database/svdb/, MIT-BIH Supraventricular Arrhythmia Database.

[17] R. O. Duda, P. E. Hart and D. G. Stork, "Pattern Classification ${ }^{2 n d}$ ed.", John Wiley \& Sons, Inc., USA, (2001).

[18] S. W. Ban, J. K. Shin and M. Lee, "Face Detection using Biologically Motivated Saliency Map Model", Proceedings of International Joint Conference on Neural Networks, (2003), July 20-24, pp.199-124.

[19] Technology Institute of Hanback Electronics, "Ubiqitous sensor network systems with ZigbeX(in Korean)", ITC Co. (2008).

[20] International Telecommunication Union, "Ubiquitous sensor networks (USN)", ITU-T Technology Watch Report \#4, Feb., (2008).

\section{Authors}

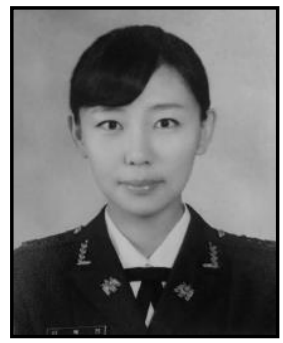

Hye-Jin Lee, received the MS degree from the Department of Information \& Communication Engineering, Dongguk University, Gyeongju, Gyeongbuk, Korea in 2013. She is currently working at Korea Army as a military officer. Her research interests include intelligent signal processing, u-health care system, neural networks, pattern recognition.

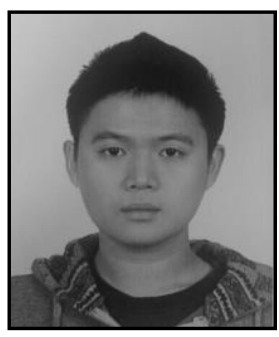

Jaeho Oh, is currently a master course student in the Department of Information \& Communication Engineering, Dongguk University, Gyeongju, Gyeongbuk, Korea. His research interests include intelligent signal processing, pattern recognition, deep learning, biologically motivated vision system.

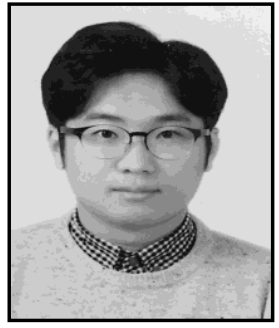

Chang-Beom Kwon, is currently a master course student in the Department of Information \& Communication Engineering, Dongguk University, Gyeongju, Gyeongbuk, Korea. His research interests include intelligent signal processing, u-health care system, pattern recognition, eye-tracking. 


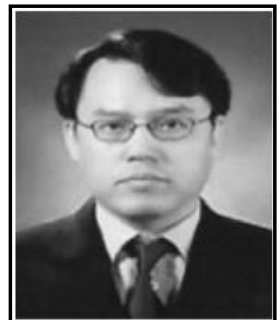

Sang-Woo Ban, received the Ph.D. degree in electrical engineering from Kyungpook National University in 2006 and is currently an associate professor in the Department of Information \& Communication Engineering, Dongguk University, Gyeongju, Gyeongbuk, Korea. His research interests include intelligent signal processing, intelligent sensor system, neural networks, pattern recognition, deep learning, biologically motivated vision system. 
International Journal of Bio-Science and Bio-Technology

Vol.8, No.1 (2016) 